\title{
Four Ways of Thinking about Information
}

\author{
Wolfgang Hofkrichner
}

\author{
wolfgang.hofkirchner@tuwien.ac.at, UTI Research Group, Vienna, Austria
}

\begin{abstract}
There are four ways of thinking: reductionism, projectivism, disjunctivism, integrativism. The gap between the "hard" science perspective and the "soft" science perspective on information reflect these ways of thinking. The paper discusses how this gap might be bridged by applying the fourth way of thinking.
\end{abstract}

Keywords: Objectivism, subjectivism, subject-object-dialectics, materialism, idealism, emergentist materialism, externalism, internalism, perspectivism

There is not yet a Science of Information. What we have is Information Science. Information Science is commonly known as something that grew out of Library and Documentation Science with the help of Computer Science, is concerned with problems in the context of the so-called storage and retrieval of information in social organisations using different media and might run under the label of Informatics as well. A Science of Information would rather be a science about information processes in natural, social and technological systems and thus have a broader scope. This is the way in which the term of Information Science is understood by a community of academics from different fields of science, engineering, humanities and arts that have been gathering around a conference series, mailing list and website with the abbreviation "FIS" (Foundations of Information Science) for more than a decade.

There is not yet a (Unified) Theory of Information. There is Information Theory which is a branch of mathematics and engineering science inaugurated by Claude E. Shannon's paper "A Mathematical Theory of Communication", published in 1948, that dealt with the problem of keeping the signal-noise-ratio in communication transmission channels under control - a problem that was faced in war experience. That which ought to be measured with his formalism was rapidly promoted to signifying "information". A (Unified) Theory of Information would have to deliberate about whether to generalise a framework that declaredly focuses on syntactical aspects and omits semantical ones when reaching out to fields in which to refer to semantics is a must. This is exactly what a lot of criticisms have been addressing since; leading to alternative theoretical approaches without ever succeeding in being recognised by a majority of the scientific community as a more general Theory of Information that would, actually, top Information Theory.

And there is not yet a unifying scientific information concept. We are accustomed to living with a multiplicity of diverse, and even contradictory, concepts of information used throughout the whole edifice of natural, social and human, and engineering sciences, not to mention everyday thinking. Attempts to unify information concepts run the risk of getting caught in reductionism or projectivism (which is, so to say, a twin of reductionism). An intriguing information concept would need to sidestep these traps.

\section{Ways of thinking}

What is a way of thinking? A way of thinking is the way how identity and difference are thought to relate to each other. Relating identity and difference may be presumed to be the most basic function of thinking. It appears in praxiological, ontological and epistemological contexts. That is, practical problems that come to thought, entities that are investigated, phenomena that have to be cognised, may be identical in certain respects but may differ from each other in other respects.

Introducing complexity, that what differs is more complex than that from which it differs, but, by the same token, it instaurates an integrated whole, and the question transforms into how the simple 
does relate to the complex, that is, how less complex problems or objects or phenomena do relate to more complex ones (Table 1).

\begin{tabular}{|l|l|l|}
\hline $\begin{array}{l}\text { ways of } \\
\text { thinking }\end{array}$ & $\begin{array}{l}\text { relationship between lower and } \\
\text { higher complexity }\end{array}$ & $\begin{array}{l}\text { unification or } \\
\text { diversification }\end{array}$ \\
\hline reductionism & $\begin{array}{l}\text { reduces higher complexity to lower } \\
\text { complexity }\end{array}$ & $\begin{array}{l}\text { unity at the cost of } \\
\text { higher complexity }\end{array}$ \\
\hline projectivism & $\begin{array}{l}\text { projects higher complexity onto } \\
\text { lower complexity }\end{array}$ & $\begin{array}{l}\text { unity at the cost of } \\
\text { lower complexity }\end{array}$ \\
\hline disjunctivism & $\begin{array}{l}\text { disjoins higher complexity from } \\
\text { lower complexity }\end{array}$ & $\begin{array}{l}\text { diversity at the cost } \\
\text { of unity }\end{array}$ \\
\hline integrativism & $\begin{array}{l}\text { integrates higher and lower } \\
\text { complexity (as well as differentiates } \\
\text { higher and lower complexity) }\end{array}$ & $\begin{array}{l}\text { unity through } \\
\text { diversity }\end{array}$ \\
\hline
\end{tabular}

Table 1: Ways of thinking

The first way of thinking, in terms of ideal types, establishes identity by eliminating the difference for the benefit of the less complex side of the difference and at the cost of the more complex side; it reduces "higher complexity" to "lower complexity"; this is known as reductionism. Reductionism is still the main stream of natural science.

The counterpart of the reductive way of thinking is what might be called "projective". Projective thinking also establishes identity by eliminating the difference, albeit for the benefit of the more complex side of the difference and at the cost of the less complex side; it takes the "higher" level of complexity as its point of departure and extrapolates or projects from there to the "lower" level of complexity. It overestimates the role of the whole and belittles the role of the parts. This is one trait of humanities. Both the reductive and the projective way of thinking yield unity without diversity.

There is a third way opposed to both of the others in that it eliminates identity by establishing the difference for the sake of each manifestation of complexity in its own right; it abandons all relationships between all of them by treating them as disjunctive; it dissociates one from the other, it dichotomises and yields dualism (or pluralism) in the sense of diversity without unity. Let's call it disjunctivism. The often bemoaned cleft between the so-called two cultures of hard science and soft science (humanities) is the most striking example for this way of thinking. In fact, this is a description of the state of the scientific adventure as a multiplicity of mono-disciplinary approaches that are alien and deaf towards each other.

And, finally, there is a fourth way of thinking that is opposed to all of the others, to reductionism, projectivism and disjunctivism as well: integrativism. This is a way of thinking that establishes identity as well as difference favouring neither of the manifestations of complexity; it establishes identity in line with the difference; it integrates both sides of the difference (yielding unity) and it differentiates identity (yielding diversity); it is a way of thinking that is based upon integration and differentiation; it is opposed to both dissociation and unification and yields unity and diversity in one. It integrates "lower" and "higher complexity" by establishing a dialectical relationship between them.

A dialectical relationship is said to exist, if the following criteria come true: firstly, both sides of the relation are opposed to each other; secondly, they depend on each other; thirdly, they are asymmetrical in that neither side can be replaced with the other without simultaneously replacing the mode of relationship. Master-and-slave or mother-and-daughter are examples for dialectical relationships. 


\subsection{Reductionism}

The relationship between practical problems, entities and phenomena of different complexity might be visualised using set-theoretical considerations as follows: be $A$ the simple and $B$ the complex. Then $A$ is the basic set, $B$ a subset (and B' the complementary set).

This means: $B$ is differentiated, given the basis $A ; B$ is different, it differs from all $A$ that is not $B$ (which is $\left.B^{\prime}\right)$; $A$ represents the identity of both sides of the difference ( $B$ and $\left.B^{\prime}\right)$.

Reduction makes the border between $B$ and $B^{\prime}$ disappear such that $B$ assumes to be $A$. Reductionism holds that every $B$ is nothing but $A$.

A praxic example: let $A$ be the knowledge about life in prehuman nature and $B$ the values for human conduct. Then reductionism would see the values as consequences of how nature is behaving. So, for instance, the "struggle for life" in nature is seen as the justification of the "struggle for life" in society.

An ontic example: let $A$ be living nature and $B$ humans. Then reductionism conjures away the difference humans make in living nature. So, for instance, ethology looks upon humans as naked apes.

An epistemic example: let A be biology and B sociology. Then reductionism tries to draw social conclusions from biological premises. So, for instance, does so-called "sociobiology" that contends that biology is the discipline that comes first.

\subsection{Projectivism}

Second, projection makes the border between $B$ and $B$ ' disappear such that $A$ assumes to be like B. Projectivism holds that every $A$ is nothing but $B$.

The praxic example: according to projectivism, values of human conduct would hold for nature too. The "struggle for life", originally found in society, is expanded to cover pre-human nature too.

The ontic example: according to projectivism, living nature does not differ from humans. Anthropomorphistic fallacies extrapolate human traits to nonhuman species and organisms like the "mind".

The epistemic example: according to projectivism, biological conclusions shall be derived from sociological premises. Ant colonies and bee hives are used to be likened to human states with queens, workers etc.

Suffice to mention that both reductionism and projectivism arrive at the equation $A=B$ but that in the first case it means $A=A$ and in the second one $B=B$.

\subsection{Disjunctivism}

Third, disjunction conjures away the common ground of $B$ and $B$ '. Disjunctivism holds that $A$ and $B$ are disjunct.

The praxic example: the realm of behaviour in nature and the realm of human conduct would have nothing in common. E.g., there is no connection whatever between natural facts and human values.

The ontic example: living nature would be extrahuman. E.g., animals don't have souls.

The epistemic example: biology and sociology would in no way depend on one another. E.g., propositions about the social life of living systems and propositions about humans in society use incompatible languages and there can be no translation.

\subsection{Integrativism}

Fourth, integration (with differentiation) sees $B$ and $B$ ' integrated into $A$ and, at the same time, $A$ differentiated into $B$ and $B^{\prime}$. $B$ is result of a differentiation in $A$.

The praxic example: human values of conduct, be it competition-oriented or cooperationoriented, cannot be derived from nature, but nature provides biotic foundations for either conduct. 
The ontic example: humans are a product of evolution of nature. In speciation they made a difference to such an extent that the new features of mind, language and work began to outbalance the dynamics of biological evolution and even change their physiological nature though humans never will be cut free from outer nature or their inner nature.

The epistemic example: sociology describes its part of reality with particular concepts and theories which relate to the concepts/theories of biology in that the latter describe spaces of possibilities for the realisation of social subject matters. Insofar a common language can be sought for.

Integrativism is itself a kind of dialectical sublation of unification and dissociation, of reductionism and projectivism and disjunctivism. A dialectical sublation eliminates the dominant role of the preceding quality rather than the quality itself. This quality is kept, that is, continued, but it is continued under the dominance of a new quality and is therefore - as Hegel liked to put it - lifted onto a next level. All of that holds for the integrativist thinking with regard to the fallacious ways of thinking. Reductionism, projectivism as well as disjunctivism are not totally negated but taken cum grano salis. Each of them has an aspect of overexaggeration that has to be abolished but, by the same token, it has an aspect that is right once the onesidedness is removed. Doing justice to these aspects is carried out through the novel integrative view - in such a way unity is established among the diverse confligating views.

Hereby the principle of unity-through-diversity is established. Either you have unity without diversity (in the first and second case of reduction and projection) or you have diversity without unity (in the third case of disjunction). Integration as viewed here yields unity in line with diversity, unity in diversity, but also diversity in unity. Diversity is considered a necessary condition of unity. Thus it is called "unity-through-diversity".

Diversity is (to be considered) a necessary, though not sufficient, condition for unity and, in turn, unity is (to be considered) a necessary, though not sufficient, condition for diversity.

Diversity and unity condition each other. Diversity can produce unity (then we speak of unitythrough-diversity), but need not to do it. Unity can enable diversity (then we speak of diversitythrough-unity), but it can constrain diversity to uniformity (then there is no unity-through-diversity).

To sum up, integrativism opposes reductionism and projectivism as well as disjunctivism but considers reduction, projection or disjunction as justified within certain boundaries when taking into account the legitimate claims of each other.

Integrativism is one step on the way to a Unified Theory of Information.

\section{Thinking in information}

The review of the classifications of information concepts/theories so far seems to support the assumption of a multitude of approaches that are diverse and irreconcilable and do not offer the possibility of consolidation. The attempt to construct order from noise to turn the babel into polyphony faces a situation which is known as "Capurro's Trilemma" between synonymity (unvocity), analogy, and equivocity of the information concepts. No option, actually, meets demands for scientificity. Synonymity does not meet them, because information in one domain would not differ from information in a different domain - a premise which has long been contested. Analogical reasoning does not meet them either, because there is no agreement on the primum analogatum, the standard of comparison. Nor does equivocity meet them, because the babel of languages which are not communicable would mean the end of scientific enterprise at all (Table 2). 


\begin{tabular}{|c|c|c|}
\hline \begin{tabular}{|l|} 
Capurro's \\
Tilemma \\
\end{tabular} & information terms... & problem \\
\hline $\begin{array}{l}\text { synonymity/ } \\
\text { univocity }\end{array}$ & ... share the same meaning & false \\
\hline analogy & ... possess similar meanings & $\begin{array}{l}\text { what is the standard of } \\
\text { comparison? }\end{array}$ \\
\hline equivocity & ... possess different meanings & Babel \\
\hline $\begin{array}{l}\text { historical and } \\
\text { logical account }\end{array}$ & $\begin{array}{l}\text {... reflect a specification } \\
\text { hierarchy that resembles the } \\
\text { becoming }\end{array}$ & $\begin{array}{l}\text { never-ending process } \\
\text { of defining and refining }\end{array}$ \\
\hline
\end{tabular}

Table 2: Capurro's Trilemma

If we take a closer look, we can discover the connection between the options given and scientific strands:

- synonymity is the option preferred by a "hard" science perspective;

- analogy goes with the "soft" science perspective; and

- equivocity seals in each of those perspectives as a separate pillar according to the so-called two-cultures perspective.

\begin{tabular}{|l|l|l|l|}
\hline $\begin{array}{l}\text { information } \\
\text { concepts }\end{array}$ & $\begin{array}{l}\text { praxiological } \\
\text { aspects }\end{array}$ & $\begin{array}{l}\text { ontological } \\
\text { aspects }\end{array}$ & $\begin{array}{l}\text { epistemological } \\
\text { aspects }\end{array}$ \\
\hline $\begin{array}{l}\text { synonymism } \\
\text { (reductionism) }\end{array}$ & $\begin{array}{l}\text { objectivism: } \\
\text { object of action }\end{array}$ & $\begin{array}{l}\text { materialism: } \\
\text { material object }\end{array}$ & $\begin{array}{l}\text { externalism: } \\
\text { objective method }\end{array}$ \\
\hline $\begin{array}{l}\text { analogism } \\
\text { (projectivism) }\end{array}$ & & subjectivism: \\
\cline { 1 - 2 } $\begin{array}{l}\text { equivocalism } \\
\text { (disjunctivism) }\end{array}$ & $\begin{array}{l}\text { subjective action } \\
\text { idealism: } \\
\text { immaterial action }\end{array}$ & $\begin{array}{l}\text { internalism: } \\
\text { action interpretative }\end{array}$ \\
\hline $\begin{array}{l}\text { historical and } \\
\text { logical account } \\
\text { (integrativism) }\end{array}$ & $\begin{array}{l}\text { subject-object } \\
\text { dialectics }\end{array}$ & $\begin{array}{l}\text { emergentist } \\
\text { materialism }\end{array}$ & $\begin{array}{l}\text { perspective- } \\
\text { shifting } \\
\text { methodology }\end{array}$ \\
\hline
\end{tabular}

Table 3: Information concepts categorization

Each of the "hard" and "soft" science and two-cultures perspectives reflects one distinctive way of thinking, that is,

- the "hard" science perspective reflects reductionism,

- the "soft" science perspective reflects projectivism, and

- the two-cultures perspective reflects disjunctivism.

What is missing so far, is integrativism. Integrativism will dissolve Capurro's trilemma.

\subsection{Information reductionism}

Reductionism reduces the meaning of information to one and the same meaning. The reductionist way of thinking information approaches information as a "hard" fact that is given to whatever science (this holds for everyday thinking too) as the one and only thing (Table 3 , line 1). 
Praxiologically, information is treated as object of practical action of humans whatsoever, i.e., in the context of social (cultural, political, economic) steering and intervention, in environmental management, in the usage and the design of technology. It is a something that can be handled, in particular, stored, retrieved, distributed, transmitted, received, processed. This is "objectivism".

Ontologically, this something is conceived as an object of the real-world, be it in the realm of human society or in the wider physical world, but it needs not necessarily to be a substance in its own right like matter is said to be in order to qualify for a materialistic ontology. It is sufficient to look upon it as a derivative of matter, that is, as a property of matter like structure is usually conceived which holds for signal transmission as well. So it's considered a material object. This is "materialism". It's worth noting that the nature of information in this context is perceived as rather fixed and static than fluid or dynamic.

Epistemologically, this material object is the object of inquiry by empirical and formal-scientific methods all of which are carried out in the so-called third-person perspective which means, from the point of view of an outside observer. Formalisms are developed for measuring the structure of matter or signals. This is "externalism".

\subsection{Information projectivism}

Projectivism projects a particular meaning of information to all the other meanings. The projectivistic way of thinking information is to approach it as a "soft" phenomenon. That is, in analogy to that subjective human action that is carried out by human actors and esteemed an information process, subjective activity of the same kind is postulated to be carried out by any agents that are said to populate the world (Table 3, line 2).

This is, praxiologically, a projection of human subjectivity in information processes onto processes with nonhuman entities the degree of subjectivity of which might be put into question "subjectivism".

This is, ontologically, a projection of a presumed intentional nature of human information processes onto the nature of processes caused by nonhuman entities the intendedness of which is questionable - "idealism". Hence the fluidity and dynamicity of all information in an idealistic view.

This is, epistemologically, a projection of subjective methods of gaining insight into the phenomenon of information in humans onto the study of nonhuman information processes - "internalism".

\subsection{Information disjunctivism}

Disjunctivism disjoins every meaning from any other meaning of information (Table 3, line 3 ). The disjunctivist way of thinking information reserves a distinct category for the subjective (human) action of information, praxiologically, in the context of a social discourse made up of humans only; ontologically, as a human phenomenon only; and, epistemologically, as subject to subjective interpretation only.

\subsection{Information integrativism}

We found an objective view with reductionism that treats information as object with an exclusive focus on the structural aspect and a subjective view with projectivism and disjunctivism that regard information as an agent's subjective action or activity with an exclusive focus on the process aspect. Integrativism reveals information as a subjective process that is constrained and enabled by information as an objective structure that is produced by information as a subjective process. Processes process structures and structures structure processes. That way the processual and the structural views are prompted to run together as much as the subjectivistic and objectivistic, idealistic and materalistic, internalistic and externalistic views are mediated and the two cultures of the "soft" and the "hard" sciences are made for bridging (Table 3, line 4). 


\subsubsection{Beyond objectivism and subjectivism}

Instead of objectivism or subjectivism, a kind of subject-object-dialectics is promoted. Objects and subjects are defined by (1) mutual exclusion as opposites (2) depending on each other (3) in an asymmetrical relation.

The subject-object dialectics can be visualised by a cycle by which the subject couples up to the object (Figure 1). On the one hand, the object bears significance to the subject in so far as its objective properties are able to suit subjective functions. On the other, the subject designates the object for serving it one way or another because it needs to reach out for that which it makes into an object and it needs to approach the object in a subjective way.

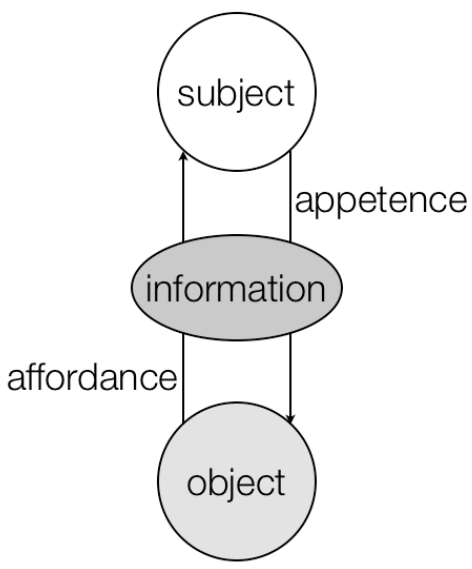

information is subjective and objective at the same time.

Figure 1: Subject-object dialectics in information

It is in this very relationship that we find the origins of information: we have subjective signification relating to objective significance.

Praxis is the ongoing process of subjecting objects to humans while factoring in inertia. However, more often than not this inertia turns out to be due not to the inert nature of the objects but to the level of subjectiveness they inhere according to their nature as kind of subjects. In that respect, humans have to come to acknowledge that they interfere with quasi- and proto-subjects and that it is up to humans, as fully-fledged subjects, to minimise the frictions occurring in the course of interaction between different kinds of subjects in order to be able to better pursue their own interests.

\subsubsection{Beyond materialism and idealism}

Instead of materialism or idealism which is monism and instead of dualism a dialectical answer is favoured.

Dialectics recognises identity and difference of matter and information at the same time: it recognises identity, given the difference, for this identity enables these different sides to interact; and it recognises the difference, given identity, for this makes it possible to differentiate matter and information as different specifications of an identical, common, genus. This common ground must be one of the two sides, since otherwise the intention of integrativism is flawed. And it should be that one of the two sides that evidences giving rise to the other, though in a non-derivative way.

Emergent(ist) materialism is known as philosophy of mind that fulfils that criterion. Matter is the common ground but leaves room for emergent properties and events as mind is regarded which is conceived to be of a different materiality compared with the simple, pure, materiality that occurs in the non-emergent state of matter. The notion of emergentist materialism shall here be taken in a wider sense to overarch all manifestations of information and not only mind.

Recalling the subject-object dialectical cycle, we have to take into consideration that a subject never relates directly to an object. Its relation to the object is always mediated. It construes the 
means of mediation. In the course of the subject's acting upon the object the subject gives rise to something new by which it mediates itself with the object - the sign. The appearance of the sign (signans) turns the subject into a signmaker (signator); the signification process (significatio) into a designation process (designatio) which means that the signification process is sign-mediated; and the object into a something (to be) signified (signandum/signatum) that bears a significance for the subject (significantia).

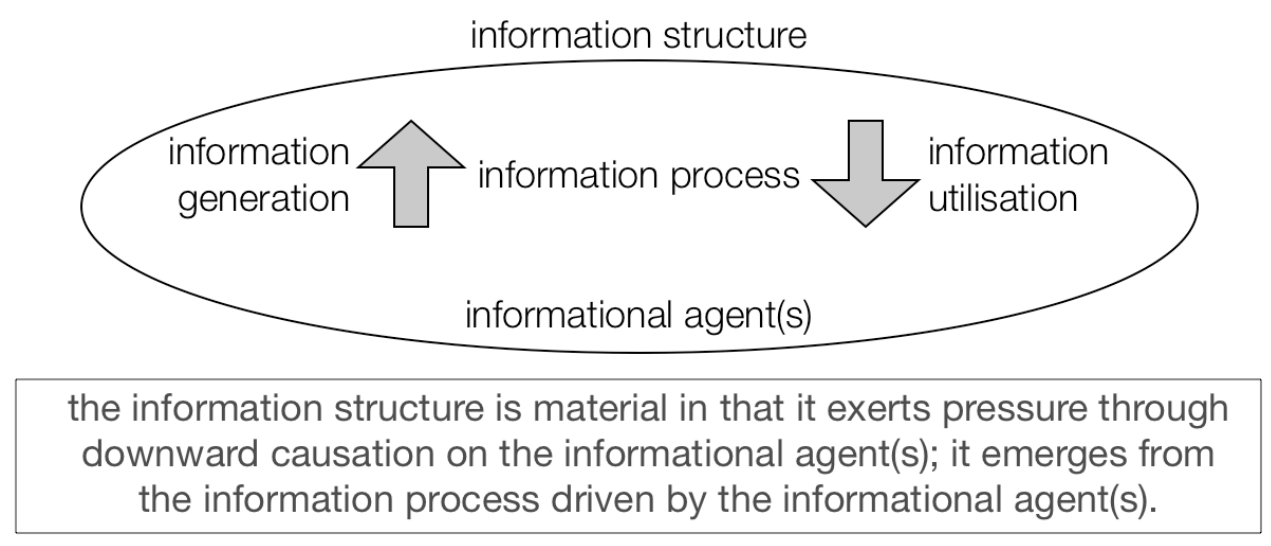

Figure 2: Emergentist materialism in information

When the signator relates to the signandum, the signator generates the signans - this is an information process by which an information structure emerges; when the signans has emerged, the signator relates to the signatum only by utilising the signans - an information process in which the information structure exerts some dominance. Thus we have an oscillation between information process and information structure (Figure 2).

Information is a relation, not a material entity, nor an ideational event. It is a mediated relation, not a direct relation between something - a subject - and another thing - an object. It is mediated by a third thing, which gives the whole relationship its unique nature.

\subsubsection{Beyond externalism and internalism}

Instead of externalism or internalism, a perspective-shifting methodology is needed.

Humanities are inclined to approach human subjects and thus human information by means of a first-person method. But there is social sciences like behavioural sciences or communication studies or cultural studies and there is psychology and cognitive sciences that approach human subjects and human information processes and structures in a third-person manner. Human subjects seem to be researchable from within and from without. And there are nonhuman subjects, quasiand proto-subjects. They seem open to inquiry only from without. But if we suggest a genealogical lineage from proto- to quasi- to fully-fledged subjects and thus a deep connection between all kinds of subjects, why should an investigation of the predecessors of human subjects from within be impossible? We just would have to accept that empathy comes stepwise and homo sapiens which disposes of the most sophisticated empathic capabilities can try to apply these capabilities in grades. We can conclude: subjects and their information processes and structures might be studied from an external and internal perspective as well. Objects turn out to be subjects that can be studied as objects and as subjects as well. Objects and subjects are two sides of the coin (Figure $3 a$ and $b)$. 


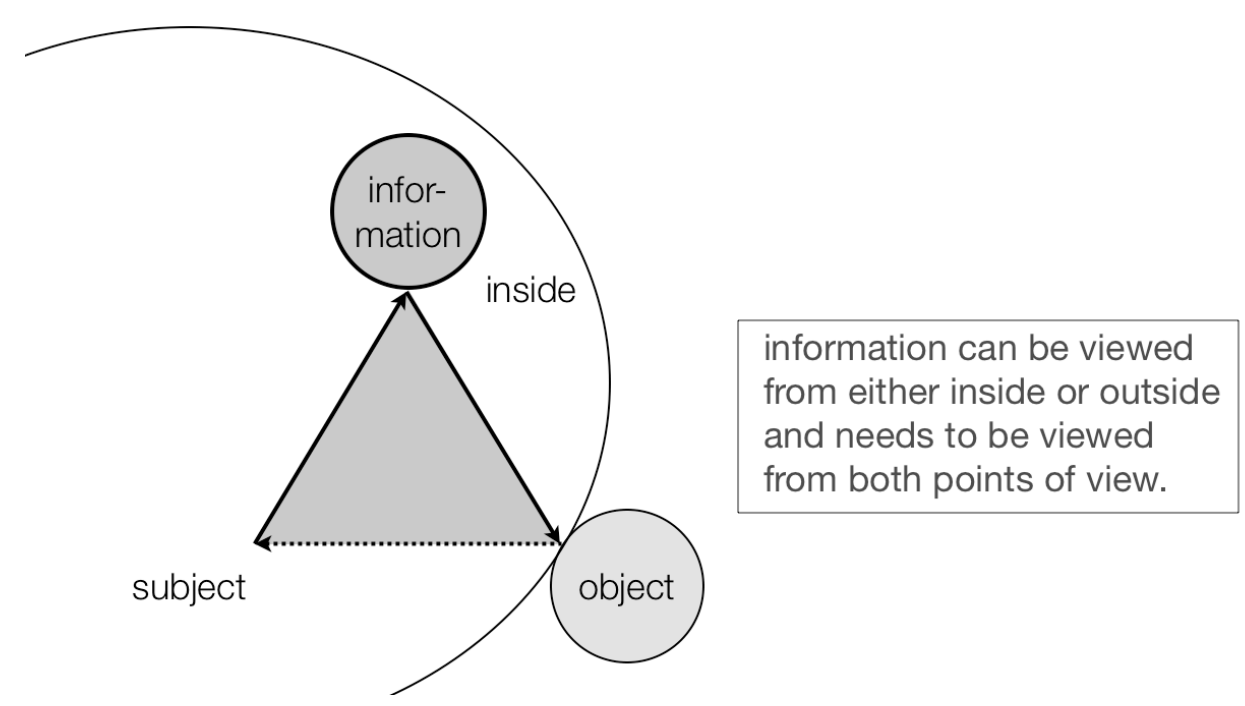

Figure 3a: Perspective-shifting methodology in information: internalist perspective

Processes and structures of information are transmuting into one another. In order to do justice to the different mutations of information shifts in the perspective taken are appropriate here too. One shift is from viewing fluid information (as a process) to viewing frozen information (as a structure). Since this structure seems to represent something that might enter an information process anew, another shift is from grasping the freeze to grasping the leaking of melted and liquified information. This would help understand the reaching out of the "potential", "bound" information (which is the structure) just by virtue of its showing up (as "free" information) to whatever is out there. A next step - the "actualisation" of the "potential" information by a subject - could be seen as a step in which the process is frozen down into "bound" information again, but finds itself in another structure, in a new structure generated by this very subject (which, in turn, might as new "potential" information become a starting point for another information process). So, one shift of perspective is preparing the ground for the next shift.

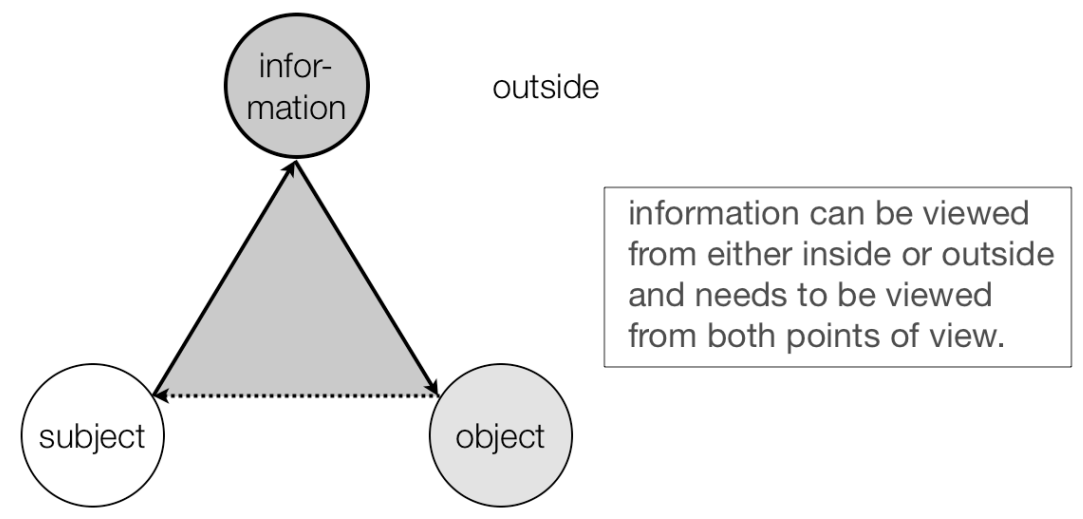

Figure 3a: Perspective-shifting methodology in information: externalist perspective

\section{Outlook: information as complex systems concept}

Having discussed the four ways of thinking and how information conceptualizations may reflect these four ways of thinking, the ground may have been prepared for a next step that applies these 
philosophy of information considerations to cross-disciplinary thinking like complex systems thinking. The author tries to do so in his forthcoming book (Hofkirchner, 2011).

\section{References}

Hofkirchner, W. (2011). Emergent information. A Unified Theory of Information Framework. World Scientific Series in Information Studies Vol.3. Singapore: World Scientific. [in print]

\section{About the Author}

Wolfgang Hofkirchner Associate Professor for Technology Assesment, Vienna University of Technology. Tries to promote a new science of information that comprises studies in information, information society and information technology. 